\title{
AUFSÄTZE
}

\section{DER BILDUNGSAUFTRAG IM JUGENDFREIWILLIGENDIENST - Theoretisch-konzeptionelle Überlegungen zur Projekt- methode als intentionaler Förderung des Nicht-Intentionalen}

\author{
Dr. Martin Nugel \\ Wissenschaftlicher Mitarbeiter am Lehrstuhl für Allgemeine Pädagogik der \\ Universität Bamberg | Referent für Freiwillige Soziale Dienste Nordbayern, \\ Nürnberg | martin.nugel@uni-bamberg.de \\ Peter März \\ Diplomsozialpädagoge | Referent für Freiwillige Soziale Dienste \\ Nordbayern, Nürnberg | p.maerz@ejn.de \\ Fabian Meissner \\ M.A. Politikwissenschaft | Verwaltungsleiter bei den Freiwilligen \\ Sozialen Diensten Nordbayern, Nürnberg | meissner.fsd@ejn.de \\ Uwe Schmidt \\ Diplomsozialpädagoge | Referent für Freiwillige Soziale Dienste \\ Nordbayern, Nürnberg | u.schmidt@ejn.de
}

\begin{abstract}
Jugendfreiwilligendienste erfüllen ihren gesetzlichen Bildungsauftrag im Spannungsfeld von persönlichkeitsbildenden und berufsorientierenden Maßnahmen. Der Beitrag beschreibt theoretische Zugänge zum Lernen in Jugendfreiwilligendiensten als Form kompetenzorientierter Alltagsbildung. Außerdem werden konzeptionelle Überlegungen zur Projektarbeit als Methode der ,intentionalen Förderung des Nicht-Intentionalen angestellt. Die Herausforderung für die Träger von Jugendfreiwilligendiensten besteht dabei darin, den Erwerb von spezifischen Handlungskompetenzen im Kontext non-formaler Bildungssettings bzw. informeller Lernprozesse zu initiieren.
\end{abstract}

Schlagwörter: Bildung, Kompetenzerwerb, Lernformen, Informelles Lernen, Alltagsbildung, Projektmethode.

The educational function of Youth Volunteer Services considering the Project-Learning-Method

Young people's voluntary services can be characterized by the poles of personal development and carreer guidance programmes. From the perspective of educational theory the challenge for providers of voluntary services is to strengthen the statutory educational responsibility ("gesetzlicher Bildungsauftrag"), as chance to form the personality und the thereby broader area of formal education. The theory of general education ('Alltagsbildung') can be of use to describe the connection of personality development and carreer guidance. From methodological perspective a specific form is needed, which is founded in the project work.

Keywords: Education, Competence-Building, Forms of Learning, Informal Learning, Project Work. 


\section{Einleitung: Der Bildungsauftrag im Jugendfreiwilligendienst}

2014 jährt sich zum 50. Mal die Einführung eines Jugendfreiwilligendienstes in der Bundesrepublik Deutschland. In seiner Sitzung vom 29. April 1964 beschloss der Deutsche Bundestag das Gesetz zur Förderung eines Freiwilligen Sozialen Jahres. ${ }^{1}$ Seitdem hat sich die Landschaft von Freiwilligendiensten erheblich ausdifferenziert. Konstitutiv ist dabei sowohl für das Freiwillige Soziale Jahr (FSJ), das Freiwillige Ökologische Jahr (FÖJ), den Bundesfreiwilligendienst (BFD) wie auch den Internationalen Jugendfreiwilligendienst (IJFD) die Charakterisierung als „Lernund Orientierungsdienst“ bzw. „Bildungsdienst“ ${ }^{2}$. Das zentrale Merkmal von Jugendfreiwilligendiensten besteht in ihrem vom Gesetzgeber formulierten Bildungsauftrag, wie er in der Neufassung des Jugendfreiwilligendienstgesetzes vom 16. Mai 2008 herausgehoben wird. Der Auftrag als Bildungsdienst liegt dementsprechend in der Förderung der „Bildungsfähigkeit der Jugendlichen“, wie es in §1 Absatz 1 JFDG $^{3}$ heißt. Der vorliegende Beitrag rekonstruiert diesen Bildungsauftrag von Jugendfreiwilligendiensten am Beispiel des Freiwilligen Sozialen Jahres.

Der Gedanke eines Bildungsauftrags, der auf die Bildungsfähigkeit der Freiwilligen zielt, lag bereits den Überlegungen bei der Einführung des Freiwilligendienstes im Jahre 1964 zugrunde. Deutlich wird dies in den Redebeiträgen zur dritten Lesung des Gesetzentwurfes im Deutschen Bundestag vom April 1964. So begründete die FDP-Bundestagsabgeordnete Liselotte Funcke den Bildungscharakter des Freiwilligenjahres mit dem Argument, dass ,[u]nsere Demokratie ... auf der freien Entscheidung zur Selbstverpflichtung aufgebaut " ${ }^{4}$ sei. Vor dem Hintergrund der Antinomie, also der Unvereinbarkeit von Gesetzen, zwischen der „Freiheit zur Selbstverwirklichung“ und dem Ruf nach „staatlichen Zwangsmaßnahmen“ (mit Blick auf ein verpflichtendes soziales Jahr) müsse es „bei der Erziehung der heranwachsenden Generation entscheidend darauf ankommen, dass die Jugend die Fähigkeit zur selbständigen Entscheidung von frühauf übt und entwickelt.“ ${ }^{5}$ Diese Entscheidung des Gesetzgebers, nach den Pflichtschuljahren und vor dem Eintritt in Berufsausbildung bzw. Berufsausübung die Möglichkeit eines freiwilligen Engagements zu gewährleisten, ist bis heute unbestritten. So kann im Kontext der Debatten um eine Präzisierung des Bildungsverständnisses von außerschulischen Angeboten der Kinder- und Jugendhilfe Bildung im Jugendfreiwilligendienst im Anschluss an den 12. Kinder- und Jugendbericht als aktiver Prozess verstanden werden, „,in dem sich

1 Protokoll der Bundestagsdebatte zur 2. und 3. Lesung des Gesetzentwurfs zur Einführung eines Freiwilligen Sozialen Jahres vom 29.4.1964 (Bundesgesetzblatt III 2160-1 vom 17.8.1964)

2 http://www.bmfsfj.de/BMFSFJ/freiwilliges-engagement.html

3 Gesetz zur Förderung von Jugendfreiwilligendiensten (Jugendfreiwilligendienstegesetz - JFDG) vom 16.05.2008 mit Änderung vom 20.12.2011.

4 Protokoll der Bundestagsdebatte zur 2. und 3. Lesung des Gesetzentwurfs zur Einführung eines Freiwilligen Sozialen Jahres vom 29.4.1964 (Bundesgesetzblatt III 2160-1 vom 17.8.1964)

5 Ebd. 
das Subjekt eigenständig und selbsttätig in der Auseinandersetzung mit der sozialen, kulturellen und natürlichen Umwelt bildet“ (BMFSFJ 2005: 83).

Der Gesetzgeber beauftragt die Jugendfreiwilligendienste dementsprechend, Ermöglichungsräume des selbst verantworteten Lernens zu schaffen und diese mit einer kontinuierlichen pädagogischen Begleitung der Freiwilligen durch die Träger und Einsatzstellen zu flankieren. ${ }^{6}$ Damit sollen soziale, kulturelle und interkulturelle Kompetenzen gefördert werden, das Verantwortungsbewusstsein für das Gemeinwohl gestärkt, die Beschäftigungsfähigkeit der Freiwilligen erhöht, sowie nicht zuletzt Prozesse der Persönlichkeitsbildung initiiert werden - eine Aufgabe, die Träger und Einsatzstellen gemeinsam verfolgen sollen. Im Anschluss an den 11. Kinder- und Jugendbericht (BMFSFJ 2002) kann der Bildungsauftrag der Jugendfreiwilligendienste als Aufgabenbereich in öffentlicher Verantwortung verstanden werden, der auf allgemeine, politische, soziale, gesundheitliche, kulturelle, naturkundliche und technische Bildung zielt. ${ }^{7}$ Das auf gesellschaftliche Teilhabe, Selbstständigkeit und Verantwortungsübernahme gerichtete Bildungsverständnis ist daher nicht nur konstitutiv für die individuelle Entwicklung, es ist auch konstitutiv für die Organisation der pädagogischen Begleitung in der subsidiären Verantwortung der Träger von Jugendfreiwilligendiensten. Dem Charakter als Bildungsjahr entspricht jedenfalls die seit den Anfängen des Jugendfreiwilligendienstes eingeforderte „pädagogische Betreuung während der Zeit des Dienstes“, so die CDU-Abgeordnete Pitz-Savelsberg in derselben Bundestagsdebatte. ${ }^{8}$ Der Bildungsgedanke wird dementsprechend dahingehend konkretisiert, dass die pädagogische Begleitung durch die Einsatzstellen und Träger an Lernzielen orientiert sein muss. ${ }^{9}$ Jugendfreiwilligendienste haben damit die Aufgabe, berufsorientierende und persönlichkeitsbildende Lernsettings zu schaffen, mit denen auf die Entwicklung entsprechender Fähigkeiten gezielt wird. Die gewünschte Bildung des Subjekts braucht folglich strukturierte und organisierte „Bildungsgelegenheiten durch eine bildungsstimulierende Umwelt und durch die Auseinandersetzung mit Personen“ (BMFSFJ 2005: 83), wie es im 12. Kinder- und Jugendbericht heißt. Der Bildungsauftrag im Jugendfreiwilligendienst bedingt daher einen „Ko-Konstruktionsprozess zwischen einem lernwilligen Subjekt und seiner sozialen Umwelt" (ebd.). Konstitutiv für den Jugendfreiwilligendienst als Bildungsdienst ist damit die Unterstützung des freiwillig engagierten und lernenden Individuums durch die professionelle Angebotsstruktur der entsprechenden Träger. In

6 Gesetz zur Förderung von Jugendfreiwilligendiensten (Jugendfreiwilligendienstegesetz - JFDG) vom 16.5. 2008, \5 Absatz 2.

7 \11 SGB VIII in der Fassung vom 26.6.1990

8 Protokoll der Bundestagsdebatte zur 2. und 3. Lesung des Gesetzentwurfs zur Einführung eines Freiwilligen Sozialen Jahres vom 29.4.1964 (Deutscher Bundestag, 4. Wahlperiode, 124. Sitzung, Bonn 29.4.1964)

9 Gesetz zur Förderung von Jugendfreiwilligendiensten (Jugendfreiwilligendienstegesetz - JFDG) vom 16.5. 2008, $\llbracket 5$ Absatz 2. 
Abgrenzung zu den Lernformen in Schule und Berufsausbildung werden Freiwillige in den Jugendfreiwilligendiensten sehr viel stärker als „Ko-Produzenten ihres eigenen Bildungsprozesses“ (ebd.) verstanden. Vor dem Hintergrund dieses Bildungsverständnisses sollte die Vielfalt und Stimulanz von Lerngelegenheiten und Lernumwelten vor allem ,an den Interessen junger Menschen anknüpfen und von ihnen mitbestimmt und mitgestaltet werden, sie zur Selbstbestimmung befähigen und zu gesellschaftlicher Mitverantwortung und zu sozialem Engagement anregen und hinführen. “ ${ }^{10}$ Der Bildungsauftrag bezieht sich also einerseits auf die Unterstützung des Aufwachsens im Sinne der direkten personenbezogenen Arbeit mit den Adressat/-innen und andererseits auf die Orientierung am anwaltschaftlichen Auftrag für die Schaffung positiver Lebensbedingungen für Jugendliche im Sinne einer rechtlich abgesicherten, zu erwartenden, bedarfsgerechten und qualifizierten Infrastruktur an entsprechenden Diensten, Angeboten und Hilfen (ebd.: 42).

Als zentrale Aufgabe für die Träger von Jugendfreiwilligendiensten kann vor diesem Hintergrund formuliert werden, dass diese an Formen des selbst regulierten und selbst verantworteten Erwerbs von Fertigkeiten, Fähigkeiten und Kompetenzen der Freiwilligen selbst anknüpfen müssen, um den intendierten Charakter als Bildungsdienst erfüllen zu können. Der folgende Beitrag geht vor diesem Hintergrund der Frage nach, wie sich der Bildungsauftrag im Jugendfreiwilligendienst erstens lern- bzw. kompetenztheoretisch begründen lässt bzw. wie selbstreguliertes Lernen im non-formalen Kontext der Einsatzstellen möglich ist (Kapitel 2). Die Frage, wie eine solche intentionale Förderung des Nicht-Intentionalen im Kontext des Jugendfreiwilligendienstes möglich ist, wird am Beispiel der Projektmethode erläutert (Kapitel 3). Zur Verdeutlichung wird ein Beispiel aus der Bildungspraxis dargestellt (Kapitel 4). Ein Ausblick auf zukünftige konzeptionelle Handlungsbzw. Forschungsbedarfe rundet den Beitrag ab (Kapitel 5).

\section{Theoretische Zugänge zu Jugendfreiwilligendiensten als Form kompetenzorientierter Alltagsbildung}

Mit dem eingangs skizzierten Verständnis zielt der Bildungsauftrag im Jugendfreiwilligendienst nicht primär auf die materielle Verwertbarkeit vordefinierter Eigenschaften und Fähigkeiten des Individuums, sondern auf das selbst-entdeckende und selbst-regulierende Potenzial menschlicher Tätigkeit. Hierzu benötigt das Individuum aber selbst wiederum bestimmte Fertigkeiten und Fähigkeiten. Theoretisch können diese Bereiche von Wissen und Können als Kompetenzen beschrieben werden, die das Individuum in die Lage versetzen, selbstbestimmt handeln zu können. Aus pädagogischer Sicht gelten diese Kompetenzen als Zielbereiche für entsprechende Lehr-/Lernprozesse.

$10 \sqrt{11}$ SGB VIII in der Fassung vom 26.6.1990 
Diese Beschreibung erfolgt im folgenden Kapitel zunächst mit dem Blick auf die Konkretisierung des Bildungsauftrags im Sinne einer allgemeinen Orientierung des Lehr/Lern-Geschehens an spezifischen Handlungskompetenzen (2.1.), in der Diskussion des Verhältnisses von Kompetenzerwerb und spezifischen Lernformen im Freiwilligenbereich (2.2.) und der Charakterisierung von engagementspezifischem Lernen als Form von Alltagsbildung (2.3.). Im Vordergrund steht hierbei die Frage, wie selbstreguliertes Lernen im non-formalen Kontext möglich ist.

\subsection{Kompetenzorientierung}

Den Referenzrahmen für den hier verwendeten Kompetenzbegriff stellt das OECD-Projekt Definition and Selection of Competencies (Definition und Auswahl von Kompetenzen, DeSeCo) dar. Die Beschreibung von Kompetenzen erfolgt dabei einerseits mit Blick auf die psychosozialen Voraussetzungen für ein individuell erfolgreiches Leben (etwa eine bezahlte berufliche Tätigkeit mit einem zum Lebensunterhalt ausreichenden Einkommen, Gesundheit und Sicherheit oder die Teilnahme am politischen Geschehen), andererseits mit Blick auf den Erfolg für die Gesellschaft im Sinne wirtschaftlicher Produktivität, demokratischer Prozesse, sozialer Kohäsion, Gleichheit und Menschenrechte bzw. ökologischer Nachhaltigkeit.

Kompetenz ist nach diesem Verständnis eine „Disposition, die Personen befähigt, bestimmte Arten von Problemen erfolgreich zu lösen, also konkrete Anforderungssituationen eines bestimmten Typs zu bewältigen“ (BMBF 2009: 72). Kompetent ist dementsprechend eine Person, die sach- bzw. sozialgerecht bestimmte Tätigkeit ausüben kann. Im DeSeCo-Projekt wird dieser Kompetenzbegriff zugrunde gelegt und als dreifache Schlüsselkompetenzen differenziert dargestellt (OECD 2005) (Abbildung 1).

Menschen sollen erstens in der Lage sein, Medien und Mittel interaktiv anzuwenden und wirksam einzusetzen (Kompetenzkategorie 1). Das können z. B. der Erwerb und die Vertiefung mündlicher und schriftlicher sprachlicher Fähigkeiten sein oder der Erwerb von Fähigkeiten zur Nutzung und Anwendung von Wissen und Informationstechnologie. Als zweiter Bereich von Schlüsselkompetenz wird der Umgang mit Heterogenität beschrieben (Kompetenzkategorie 2). Relevante Fähigkeiten sind hier der Aufbau von tragfähigen Beziehungen, die Fähigkeit zur Zusammenarbeit und Kooperation sowie die Bewältigung und die Lösung von Konflikten. Und drittens sollen Menschen befähigt sein und werden, „Verantwortung für ihre Lebensgestaltung zu übernehmen, ihr Leben im größeren Kontext zu situieren und eigenständig zu handeln“ (ebd.: 7) (Kompetenzkategorie 3). Der DeSeCo-Kompetenzrahmen nennt dabei ausdrücklich die Fähigkeit, „Lebenspläne und persönliche Projekte zu gestalten und zu realisieren“ (ebd.: 17). 
Abbildung 1: Kompetenzkategorien im DeSeCo

KOMPETENZ-

KATEGORIE 1

$\rightarrow$ Interaktive

Anwendung von

Medien und Mitteln

KOMPETENZ-

KATEGORIE 2

KOMPETENZ-

$\rightarrow$ Integrieren in heterogenen

KATEGORIE 3

Gruppen

$\rightarrow$ Eigenständiges

Handeln

Quelle: OECD 2005: 7, eigene Darstellung

Solche Schlüsselkompetenzen sind deshalb immer auf die individuelle Handlungskompetenz bezogen (Kultusministerkonferenz 2007). Unter Handlungskompetenz wird die „Bereitschaft und Befähigung des Einzelnen“ verstanden, „sich in beruflichen, gesellschaftlichen und privaten Situationen sachgerecht durchdacht sowie individuell und sozial verantwortlich zu verhalten“ (ebd.: 10). Sie entfaltet sich in den Bereichen von

- Sach- bzw. Fachkompetenz als „Bereitschaft und Befähigung, auf der Grundlage fachlichen Wissens und Könnens Aufgaben und Probleme zielorientiert, sachgerecht, methodengeleitet und selbstständig zu lösen und das Ergebnis zu beurteilen" (ebd.: 11);

- Sozialkompetenz als „Bereitschaft und Befähigung, soziale Beziehungen zu leben und zu gestalten, Zuwendungen und Spannungen zu erfassen und zu verstehen sowie sich mit Anderen rational und verantwortungsbewusst auseinander zu setzen und zu verständigen. Hierzu gehört insbesondere auch die Entwicklung sozialer Verantwortung und Solidarität“ (ebd.);

- Selbstkompetenz als „Bereitschaft und Befähigung, als individuelle Persönlichkeit die Entwicklungschancen, Anforderungen und Einschränkungen in Familie, Beruf und öffentlichem Leben zu klären, zu durchdenken und zu beurteilen, eigene Begabungen zu entfalten sowie Lebenspläne zu fassen und fortzuentwickeln. Sie umfasst Eigenschaften wie Selbstständigkeit, Kritikfähigkeit, Selbstvertrauen, Zuverlässigkeit, Verantwortungs- und Pflichtbewusstsein. Zu 
ihr gehören insbesondere auch die Entwicklung durchdachter Wertvorstellungen und die selbstbestimmte Bindung an Werte“ (ebd.).

Die OECD-Definition nennt darüber hinaus noch das reflexive Denken und Handeln als grundlegenden Bestandteil dieses Kompetenzrahmens (OECD 2005). Reflexivität erfordere komplexe Denk- und Handlungsprozesse und verlange, „dass das Subjekt zum Objekt des Denkens wird“ (ebd.: 10). In diesem Verständnis geht es also darum, nicht nur bestimmte Tätigkeiten zu erlernen und auszuführen, sondern darüber zu reflektieren, sie mit anderen Aspekten abzugleichen und evtl. abzuändern oder anzupassen. Reflexivität setzt demzufolge die Anwendung „metakognitiver Fähigkeiten (Denken über das Denken), Kreativität und eine kritische Haltung voraus“ (ebd.).

Ein solches Verständnis von reflexiver Handlungskompetenz ermöglicht es, den Bildungsauftrag der Jugendfreiwilligendienste im Hinblick auf Persönlichkeitsentwicklung und Berufsorientierung zu konkretisieren. Im freiwilligen Engagement sollen Schlüsselkompetenzen erworben bzw. verfestigt werden, die für alle Lebensbereiche wichtig, d.h. übergreifend bzw. transversal sind. Jugendfreiwilligendienste beziehen sich dabei zwar auf bereichsspezifische Dispositionen, also auf einen „begrenzten Sektor von Kontexten und Situationen“ (Klieme 2004: 11), allerdings kann davon ausgegangen werden, dass diese spezifischen Kompetenzen begrenzt verallgemeinerbar sind. Die Einübung solcher Schlüsselkompetenzen soll jungen Menschen also helfen, „wichtige Anforderungen unter verschiedenen Rahmenbedingungen zu erfüllen“ (OECD 2005: 6).

\subsection{Informelles Lernen im non-formalen Kontext}

Dieser Bildungsauftrag wird im Folgenden aus Sicht der Jugendlichen bzw. jungen Erwachsenen als informeller Lernprozess in non-formalen Lernsettings beschrieben. Zugrunde gelegt ist dabei ein Verständnis von Lernen und Bildung, wie es etwa der Streitschrift ,Zukunftsfähigkeit sichern! - Für ein neues Verhältnis von Bildung und Jugendhilfe' des Bundesjugendkuratoriums (BMFSFJ 2001), der Schrift ,Konzeptionelle Grundlagen für einen Nationalen Bildungsbericht (Rauschenbach u. a. 2004), dem 12. Kinder- und Jugendbericht (BMFSFJ 2005) oder der Aachener Erklärung (Deutscher Städtetag 2007) zugrunde liegt. Im Anschluss daran können formale und non-formale Bildungsorte bzw. formelle und informelle Lernprozesse unterschieden werden. Erfolgt die Differenzierung im ersten Fall anhand der Art und Weise der Institutionalisierung bzw. Qualität von Lernsettings, stehen im zweiten Fall die individuellen Lernprozesse im Fokus (Rohlfs u. a. 2014; Wischmeier/Macha 2012).

Formale Bildungsorte können als strukturierte Lernsettings verstanden werden. Das Lernen ist institutionengebunden und durch qualifiziertes Fachpersonal 
begleitet. Formelles Lernen ist systematisch in Bezug auf Lernziele, -zeit oder Lernförderung hin organisiert, es erfolgt aus Sicht des Lernenden zielgerichtet und ist auf den Erwerb spezifischer Kompetenzen ausgerichtet. Die Intentionalität des Lehrens folgt dabei einer rationalen Planungslogik, sie ist ergebnisorientiert, wissenschaftlich abgesichert und führt zu zertifizierten Abschlüssen. Im Vordergrund steht der Aspekt des überprüfbaren Kompetenzerwerbs, weswegen der messbare Nachweis von Leistung eine große Rolle spielt. Im Kontext der Jugendfreiwilligendienste gibt es weder formale Bildungsorte noch formelle Lernprozesse im eigentlichen Sinn. Gleichwohl spielen die Erfahrungen der Freiwilligen mit solchen Lernformen in der Schul- und/oder der Berufsausbildung eine nicht zu unterschätzende Rolle.

Der Begriff ,non-formale Bildungsorte‘ kann als „Sammelbezeichnung für alle Formen des Lernens“ verstanden werden, „die in der gesamten Umwelt außerhalb des formalisierten Bildungswesens stattfinden" (Wischmeier/Macha 2012:115). Lernen findet demzufolge gerade an solchen Orten statt, die explizit dafür gar nicht institutionalisiert worden sind. In Bezug auf Lernziele, -zeit oder Lernförderung ist dieses Lernen nicht schulisch strukturiert, eher beiläufig, implizit, unbewusst und führt nicht zu Zertifizierung. Lernprozesse erfolgen hier sehr viel häufiger unbegleitet und selbst reguliert im Alltag der Lernenden. Dabei werden „sowohl das unbeabsichtigte beiläufige Lernen wie auch ein bewusstes absichtliches Lernen in der außerschulischen Umwelt umfasst, wobei die Übergänge zwischen beiden Spielarten in der Praxis fließend sind“ (ebd.).

Mit dieser Unterscheidung ist ein zentraler Unterschied im Bildungsverständnis von Jugendfreiwilligendiensten im Vergleich mit schulischen Bildungsprozessen benannt. Bildungsprozesse und Bildungsangebote von Jugendfreiwilligendiensten sind nicht notwendigerweise mit dem Leistungsgedanken im Sinne einer Übereinstimmung mit bestimmten inhaltlichen Leistungsanforderungen verbunden, die es zu erfüllen gilt, um ein freiwilliges Jahr zu ,bestehen'. Vielmehr spielt die Freiwilligkeit des Lernens eine entscheidende Rolle. Der Unterschied liegt dabei vor allem im unterschiedlichen Verständnis der Subjekte des Lernprozesses. Jugendfreiwilligendienste betrachten Jugendlichen bzw. junge Erwachsene „nicht nur als Adressaten schulischer Bildungsprozesse, sondern als lernende Individuen, die in der Lage sind, sich Wissen und Kompetenzen selbständig, d.h. abseits des schulisch organisierten Lernens, anzueignen“ (ebd.: 116). Dieses Verständnis von informellen Lernprozessen im non-formalen Kontext, d. h. das selbst gesteuerte und selbst regulierte Lernen im alltäglichen Betrieb der Einsatzstellen bzw. in den vom Gesetzgeber vorgesehenen Form der pädagogischen Begleitung durch Bildungstage, spielt für die Kompetenzorientierung im Bildungsverständnis von Jugendfreiwilligendiensten eine entscheidende Rolle. 
Aus dieser Unterscheidung ergibt sich die Notwendigkeit, Fragen des Bildungsauftrags als Kompetenzerwerb mit den informellen Lernformen im freiwilligen Engagement und ihren non-formalen Settings zu verbinden. Kompetenzerwerb im Freiwilligenengagement erfolgt dementsprechend eben nicht in formellen Lehr-/Lernprozessen, sondern beinhaltet vor allem Formen informellen Lernens, die dem Charakter als Selbstbildung Rechnung tragen. Lernen erfolgt also stark selbstreguliert, es orientiert sich am Individuum, beinhaltet eine umfassende Sichtweise des lernenden Individuums und beschreibt solche Dispositionen, die hauptsächlich in der Realisierung evaluierbar sind, aber nicht unbedingt direkt überprüfbar (Arnold/Schüßler 2001).

Solche Lernformen können als engagementspezifisch charakterisiert werden (Düx u. a. 2009). Der Kompetenzerwerb ist dabei an informelle Lernprozesse geknüpft und erfolgt in non-formalen Einrichtungen ohne spezifischen bzw. prioritären Bildungsauftrag. ${ }^{11}$ Engagementspezifisches Lernen erfolgt also vor allem „durch die aktive Übernahme und Verantwortung in der konkreten Praxis in Ernst- und Echtsituationen“ (ebd.: 267). Entsprechende Lernprozesse sind also eher selbst verantwortet und selbst reguliert. Festgehalten werden kann, dass aufgrund der selbst verantworteten Entscheidung für ein freiwilliges Engagement solche Lernprozesse den eigenen Interessen der Freiwilligen wohl sehr nahe kommen. Die Frage ist dabei allerdings, inwieweit Lernen jenseits der Übernahme von Handlungsroutinen im alltäglichen Betriebsablauf in „,selbstbestimmter Form und mit selbst gewählten Inhalten“ (ebd.) überhaupt möglich ist bzw. inwieweit Freiwillige dazu angeregt werden können?

\subsection{Alltagsbildung durch Verantwortungsübernahme}

Das engagementspezifische Lernen als der Form informellen Lernens im Jugendfreiwilligendienst ist auf den alltäglichen Ablauf der Handlungsroutinen in den Einsatzstellen gerichtet. Theoretisch kann dieser Lernkontext mit dem Konzept der Alltagsbildung beschrieben werden (Rauschenbach 2007). Diese Theoriefigur bezeichnet gewissermaßen die „lebensweltliche Seite der Bildung“ (Rauschenbach 2011: 38). Alltagsbildung ist „das unhintergehbare Gesamtbildungsprojekt jeder einzelnen Person (...), das von vornherein kein Thema ausblendet, keine Gelegenheit auslässt und keinen Ort ausschließt" (ebd.: 41). Rauschenbach entwickelt das Konzept vor dem historischen Hintergrund der Expansion schulischen Lernens. Seit dem 19. Jahrhundert ${ }^{12}$ „schrumpfen (...) sämtliche Bildungsorte und

11 Das sind entweder Einrichtungen, die per se einen pflegerischen oder unterstützenden Charakter haben (etwa in der Alten- und Behindertenhilfe) aber keinen Bildungsauftrag verfolgen. Andererseits nehmen auch die expliziten Bildungseinrichtungen (etwa in der Jugendarbeit oder dem Elementarbereich) die Freiwilligen nicht als ,Lernende bzw. zu Bildende' wahr, die spezifische und formale Lehr-/Lernprozesse durchlaufen müssen.

12 Rauschenbach spricht von einer bildungspolitischen Revolution, die mit der Etablierung der Schulpflicht und dem Ausbau des Schulwesens verbunden ist. 
Lernwelten jenseits der Schule (...) in Anbetracht der Prägekraft dieser Bildungsinstanz zu marginalen Bildungsepisoden“ (Rauschenbach 2007: 441). Sicherlich ist diese Marginalität durch die politische Absicht der Freiwilligendienste und ihre wenngleich auch arbeitsmarktneutrale, aber doch trägerspezifische Notwendigkeit zum Erhalt der Qualitätssicherung in den verschiedenen sozialen Einrichtungen im Vergleich zu anderen Feldern der außerschulischen Jugendbildung weniger ausgeprägt. Die alltagspraktische Herausforderung bleibt jedoch bestehen - gerade angesichts der Tatsache, dass Freiwillige nicht nur in Einzelfällen von Einsatzstellen als billige Arbeitskräfte verstanden werden und die Kosten in die Bildung der Freiwilligen so gering wie möglich zu halten versucht werden. Die Herausforderung für die Freiwilligendienste besteht entsprechend darin, Bildung neben bzw. nach der Schule nicht nur als „Residualkategorie“ (ebd.: 444) Preis zu geben, sondern als Möglichkeit der persönlichkeitsbildenden, und damit umfassenden allgemeinbildenden Bereiche menschlichen Lernens und Handelns zu stärken. Eine solche allgemeinbildende Alltagsbildung ist dementsprechend

- auf das Elementare bezogen, d.h. auf einfache und grundlegende Sachverhalte, die über sich hinausweisen (Beispiele für solche elementaren Lernprozesse sind pflegende, helfende und unterstützende Tätigkeiten und die damit verbundene Erfahrung von Nähe und Distanz);

- auf das Fundamentale als Grunderfahrung und Grundeinsicht der Wahrnehmung der Welt gerichtet (z. B. die Einübung und Ausgestaltung von anderen Rollenmodellen als dem Schüler-Lehrer-bzw. Kind-Eltern-Verhältnis) und

- am Exemplarischen als dem Typischen orientiert, das für eine große Auswahl eines Sachgebiets mit gleicher Struktur steht (etwa die Übernahme einer bestimmten Position in einem sozialen Arbeitsgefüge oder die Bewältigung von Belastungen am Arbeitsplatz).

Mit dem Konzept der Alltagsbildung beschreibt Rauschenbach die, andere Seite der Bildung' (Otto/Rauschenbach 2008) jenseits standardisierter und curricular organisierter Lehr-/Lernprozesse. Ausgegangen werden kann daher davon, dass der Kompetenzerwerb im informellen bzw. non-formalen Kontext des Jugendfreiwilligendienstes quasi ,alltäglich', ,nebenbei‘ und in hohem Maße ,en passant' erfolgt, gewissermaßen als ,training on the job.$^{13}$ Lernprozesse verlaufen in den Einrichtungsstellen eher indirekt, implizit und nicht unbedingt intendiert. Von besonderer Bedeutung ist dabei, dass Einsatzstellen zwar als Bildungsorte bezeichnet werden können, diese aber dem Bildungssystem formal gerade nicht zugeordnet sind. Obwohl in ihnen „unübersehbar gelernt wird“ (Rauschenbach 2007: 444), ist es

13 Standardisiertes Lernen findet sich in vielen Einsatzstellen daher im Grunde genommen nicht bzw. nur insoweit, als die unmittelbaren Interessen der Einsatzstellen (etwa nach Arbeitssicherheit) erfüllt sein müssen. 
nicht Aufgabe der Einsatzstellen dieses Lernen zu koordinieren und zu strukturieren. Diese Aufgabe kommt der pädagogischen Begleitung durch die Träger zu. ${ }^{14}$

Im Hinblick auf Fragen der Kompetenzentwicklung sollte beim engagementspezifischen Lernen im Jugendfreiwilligendienst diese Alltagsstruktur besondere Berücksichtigung finden. Gerade für die Schlüsselkompetenz 2 und 3 ,Interagieren in heterogenen Gruppen' bzw. ,eigenständiges Handeln' stellt sich die Frage, wie die ohne Zweifel ablaufenden Lernprozesse mit der pädagogischen Begleitung verzahnt werden können, ohne dass deren informeller, nicht-intentionaler, selbstbildender Charakter unterlaufen wird.

Mit dem Konzept der Alltagsbildung rückt darüber hinaus die autobiographische Dimension in den Vordergrund (Langewand 2002). Das Verstehen des eigenen (Arbeits)-Lebens, das Finden von Sinn im eigenen (beruflichen) Handeln sind zentrale Elemente reflexiver Kompetenz. Die Kompetenzziele einer solchen Alltagsbildung können dahingehend beschrieben werden, dass junge Menschen lernen, sich in ihren Stärken und Schwächen erleben und reflektieren zu können, sodass sie einerseits in bestehende soziale Strukturen der Arbeitswelt hineinwachsen sollen, andererseits sich in diesen aber auch ausprobieren und (selbst) finden sollen. Diese autobiographische Dimension der Alltagsbildung verweist darauf, dass informelles Lernen und Kompetenzerwerb sehr stark in den ,subjektiven Interpretationen der Individuen“ (ebd.: 40) verankert ist. Dementsprechend ist es wichtig, dass im Jugendfreiwilligendienst Tätigkeiten erlernt und routinisiert werden können, die für die Freiwilligen eine gute und sinnvolle Bedeutung und Wert in sich haben. Und nicht zuletzt geht es darum, im eigenen, bildenden Tun, Selbstwirksamkeit zu erfahren und somit ein Stück weit eine positive Identität aufbauen zu können.

Wenn standardisierte Formen des Lernens angesichts des skizzierten Bildungsverständnisses aber nicht etabliert werden können, dann gilt es umso mehr danach $\mathrm{zu}$ fragen, wie die bereits existierenden alltäglichen Lern- und Bildungserfahrungen so aufbereitet werden können, dass Lernerfahrungen explizit gemacht und zu bleibenden Bildungserfahrungen erweitert werden können. Anders gefragt: Wie kann persönliche und berufliche Entwicklung gerade in den stark vorstrukturierten Feldern der sozialen Systeme erfolgen? Wie ist der Erwerb von engagementspezifischen Schlüsselkompetenzen, wie sie im DeSeCo-Kompetenzbereich 2 und 3 beschrieben werden, im Kontext der jeweiligen Organisation möglich?

14 Dieses ,bildende Lernen' (Weber 1999) weist allerdings ebenfalls formalisierte Strukturen auf. So gibt es eine gesetzliche Grundlage, das Bildungsverhältnis ist durch ein bestimmtes Rollenverhältnis gekennzeichnet (Bildungsreferent-Freiwillige-Einsatzstelle/Anleiter), es gibt bestimmte Lernorte (Seminar und Arbeitsstelle) und Anforderungen an alle Beteiligten (Qualitätshandbuch für Träger; Arbeitsverträge für Freiwillige; Konzeptionen für Einsatzstellen usw.). 
Diese Fragen beziehen sich unmittelbar auf das didaktische Arrangement von kompetenzorientierten Lernprozessen in den quasi alltäglichen, nicht-formalen Lernwelten und eher informellen Lernprozessen in den jeweiligen Einsatzstellen. Gefragt werden muss daher, wie angesichts des Charakters des Freiwilligenlernens als informeller, engagementspezifischer Lernform in non-formalen Bildungssettings eine intentionale Förderung des Nicht-Intentionalen gelingen kann?

\section{Projektarbeit als Methode der, intentionalen Förderung des Nicht-Intentionalen ${ }^{15}$}

Im Folgenden wird als eine mögliche Methode zur Umsetzung der intentionalen Förderung des Nicht-Intentionalen die Methode der Projektarbeit vorgestellt (Frey 2012/1982). Projektarbeit im Jugendfreiwilligendienst zielt dabei auf die Förderung reflexiver Handlungskompetenz, insbesondere auf die Kompetenzkategorien 2 und 3 im DeSeCo (OECD 2005: 7). Die Freiwilligen sollen einerseits die Interaktion in heterogenen Gruppen einüben und andererseits eigenständiges Handeln in Ernst- und Echtsituationen trainieren. Die Auseinandersetzung mit der Projektinitiative, die Auswahl des endgültigen Projektauftrages sowie Planung, Durchführung und Evaluation bestimmter Themen- und Aufgabenstellungen werden dabei als bildende Elemente des individuellen Kompetenzerwerbs verstanden. Die Projektmethode macht also gewissermaßen aus dem „üblichem Tun“ im Alltag ein „bildendes Tun“ (Frey 2012/1982: 13). Als Lernziel wird die Fähigkeit anvisiert, Verantwortung für den jeweiligen Arbeitsbereich bzw. die entsprechende Arbeitsaufgabe zu übernehmen und Problemstellungen eigenständig zu bearbeiten. In diesem Sinne zielt Projektarbeit auf die Kompetenz, die eigene Person im Kontext der strukturierten Arbeitsprozesse in der Einsatzstelle zu deuten und diesen einen eigenen Sinn und Zweck zu geben. Damit ist der Bezug zum Kompetenzbereich 3 des DeSeCo hergestellt, in dem es ausdrücklich um die Fähigkeit geht,

- „ein Projekt zu definieren und Ziele zu setzen

- die verfügbaren wie auch die benötigten Ressourcen (z. B. Zeit und Geld) festzulegen und zu evaluieren

- Ziele zu präzisieren und Prioritäten zu setzen

- erforderliche Ressourcen zur Erreichung mehrerer Ziele einzusetzen

- aus vergangenen Handlungen zu lernen und zukünftige Ergebnisse zu planen

15 In den beiden folgenden Kapiteln 3 und 4 werden Erfahrungen mit der Projektmethode im Rahmen der Seminararbeit durch die Freiwilligen Sozialen Dienste Nordbayern reflektiert. Die Materialien bzw. Beispiele stammen aus der Pilotphase des Projekts in den Jahrgängen 2012/13 bzw. 2013/14. Einige Beispiele solcher Projekte sind in der Broschüre „Es kommt nicht darauf an, wie alt man wird, sondern wie man alt wird“ (Zitat von Prof. Dr. Ursula Lehr, ehem. Bundesfamilienministerin, Vorsitzende der Bundesarbeitsgemeinschaft der Senioren-Organisationen, BAGSO) dargestellt (Freiwillige Soziale Dienste Nordbayern der Evangelischen Jugend Nürnberg (2013) (http://fsd.ejn.de/wp-content/uploads/Broschuere-Altenhilfe-PDF.pdf). 
- Fortschritte zu überwachen und im Verlauf des Projekts nötige Korrekturen vorzunehmen“ (OECD 2005: 17).

Die Projektarbeit zielt auf ein Kompetenzverständnis, das über die einfache Wiedergabe angesammelten Wissens, die bloße Übernahme von Verhaltensroutinen oder die Erreichung bestimmter kognitiver Kompetenzniveaus hinausgeht. Sie bezieht sich auf die bei den Freiwilligen „verfügbaren oder durch sie erlernbaren kognitiven Fähigkeiten und Fertigkeiten, um bestimmte Probleme zu lösen“, ohne „die damit verbundenen motivationalen, volitionalen und sozialen Bereitschaften und Fähigkeiten“ auszuklammern, die notwendig sind, ,um die Problemlösungen in variablen Situationen erfolgreich und verantwortungsvoll nutzen zu können“ (Weinert 2001: 27f.). Kompetenz äußert sich auf individueller Seite in Performanz, also der tatsächlich erbrachten Leistung. Die Entwicklung und Förderung von Kompetenz sollte daher ,eine ausreichende Breite von Lernkontexten, Aufgabenstellungen und Transfersituationen umschließen“ (Klieme u. a. 2003: 74) und sich „auf konkrete Anforderungssituationen beziehen“ (ebd.: 73). Auf institutioneller Seite gilt es die unterschiedlichen Kompetenzbereiche zu vernetzen, also die Absichten und Willen der Handelnden zu berücksichtigen aber auch ihre Erfahrung und Motivation. ${ }^{16}$ Informelle Lernprozesse sollten daher durch formelle Lehr$b z w$. Unterstützungsprozesse von Seiten der Anleitung vor Ort bzw. der Bildungsreferate der Träger gerahmt werden. Von besonderer Bedeutung ist dabei, dass Aufgaben- und Themenstellung aus dem Erfahrungs- und Bewältigungsbereich der Freiwilligen stammen sollten. So kann sowohl Unter- als auch Überforderung vermieden und der Projektauftrag letztlich bewältigt werden. Die Bedürfnisse, Neigungen und Interessen der Freiwilligen spielen dabei eine zentrale Rolle. Eine der zentralen Reflexionsaufgaben ist daher, die eigenen Bedürfnisse und Interessen mit den Bedürfnissen der jeweiligen Zielgruppen bzw. den strukturierenden Elementen von Einsatzstellen und Träger auszugleichen.

Der Kompetenzerwerb erfolgt dabei in zwei unterschiedlichen Lernsettings. Einerseits handeln Freiwillige in ihrer Einsatzstelle in Auseinandersetzung mit den jeweiligen Zielgruppen bzw. den Teamkollegen und -kolleginnen, andererseits reflektieren sie ihre Lernschritte, Erfolge bzw. Misserfolge in der Seminargruppe. Das Lernen erfolgt dabei in mehreren, zusammenhängenden Arbeitsschritten. Am Beginn des Projekts steht die Projektinitiative, die vom Träger ausgeht und intentionalen Charakter hat. Auf dem Einführungsseminar wird die Projektmethode

16 Hartig plädiert allerdings dafür, kognitive und motivationale Bereiche für den Kontext der empirischen Erfassung von Kompetenz voneinander zu trennen. Nur so könne die wechselseitige Beziehung zwischen beiden Dimensionen untersucht werden. „Die Trennung von kognitiven und motivationalen Faktoren erlaubt Aussagen wie ,Die Mitarbeiter verfügen zwar über die notwendigen Kompetenzen, sind aber kaum motiviert' oder ,Sie sind zwar hoch motiviert, aber inkompetent'. Eine Integration der Motivation in das Kompetenzkonzept würde derartige Aussagen unmöglich machen. Personen wären nur dann als ,kompetent' zu bezeichnen, wenn sie über die kognitiven Leistungsvoraussetzungen verfügen würden und zugleich motiviert wären“ (Hartig 2008: 17). 
vorgestellt und in die Jahresplanung übernommen. Damit wird eine offene Ausgangssituation hergestellt, d.h. welche konkrete Durchführung des Projekts sich aus dem Projektauftrag ergibt, ist offen. Diese Offenheit des Lernprozesses und der geringe Strukturierungsgrad des Lehrprozesses stellen eine zentrale und notwendige Voraussetzung für den Kompetenzerwerb im oben beschriebenen Sinne dar. Denn die Freiwilligen müssen sich ja erst Klarheit darüber verschaffen, „ob und in welcher Form sie die Projektinitiative aufgreifen" (Frey 2012/1982: 56). Die Projektinitiative selbst ist also noch „ohne Bildungswert“ (ebd.). Hierin liegt das Spannungsfeld, das die Projektmethode beinhaltet. Denn inwiefern die Projektinitiative zu einem „pädagogisch wertvollen Sachverhalt“ (ebd.) führt, das lässt sich nicht ohne weiteres und von vornherein festlegen, sondern basiert auf den Vorerfahrungen der Freiwilligen und den Möglichkeiten der Einsatzstelle. Gerade für die pädagogische Begleitung durch die Seminarleitungen ergibt sich hier eine besondere Herausforderung, sind doch grundsätzlich „alle Erscheinungen unseres Lebens sowie der natürlichen und hergestellten Umwelt würdig, Gegenstand einer Projektinitiative zu werden“ (ebd.). Der Gegenstand des Projekts, die Art und Weise der Auseinander- und Umsetzung, die Intensität sind daher nicht vorab festlegbar, zumindest dann nicht, wenn man verschultes und curriculares Lernen als unvereinbar mit dem Freiwilligendienst ansieht. Mit anderen Worten: Die Projektinitiative wird für die Freiwilligen ,erst allmählich zur Bildung, indem sie sich mit ihr in einer bestimmten Weise auseinandersetzen ... und zu einem Betätigungsgebiet entwickeln“ (ebd.: 56). Gleichwohl benötigt diese Auseinandersetzung aber einen vorab vereinbarten Rahmen.

In einem weiteren Schritt erfolgt daher auf dem ersten Zwischenseminar eine Einführung in das Thema Projektmanagement. Als Bestandteil des Projektes werden die Auseinandersetzung mit der Projektinitiative, die Auswahl des endgültigen Themas sowie die gemeinsame Entwicklung des Betätigungsgebietes in Absprache von Freiwilligen, Einsatzstelle und Träger thematisiert. Abschließend werden die Ideen von den Freiwilligen anhand eines schriftlich fixierten und verbindlichen „Projektauftrags“ konkretisiert (Abbildung 2). In der Zwischenzeit bis zum nächsten Seminar sprechen sich die Freiwilligen mit ihren Anleitungen in den Einsatzstellen über die Umsetzbarkeit ab, entwerfen ggf. zusammen Alternativen und beschließen die letztendliche Projektidee in enger Rückkoppelung mit den Seminarreferenten und -referentinnen.

Der Stand der Vorbereitungen wird dann im weiteren Seminarverlauf ebenso reflektiert wie bei den Einsatzstellenbesuchen. Auf dem zweiten und/oder dritten Zwischenseminar erfolgt dann eine Zwischenbilanz in der Seminargruppe. Der Vordruck „Projektbericht“ wird durchgesprochen und mitgegeben. Die Bearbeitung wird verbindlich terminiert bis spätestens vier Wochen vor dem letzten Seminar. Spätestens ab dieser Zeit sollten die Projekte durchgeführt werden. Am Abschluss-Seminar erfolgt dann von den Freiwilligen eine kurze Präsentation für 


\title{
die Gruppe wie auch anhand des Projektberichts eine Dokumentation für diverse Öffentlichkeitsarbeitsbereiche.
}

\author{
Abbildung 2: Beispielhafte Darstellung eines schriftlichen Projektauftrags an die \\ Freiwilligen
}

\section{PROJEKTAUFTRAG}

Name:

Einsatzstelle:

AUFGABE: Im Laufe des FSJ-Jahres erarbeitet der/die Freiwillige an/mit der Einsatzstelle ein Projekt. Hierzu gehören die Planung, die Durchführung sowie die Präsentation.

THEMENFINDUNG: Hilfestellung: Konzeption der Einsatzstelle, Jahresberichte, Protokolle anderer Projekte, Fachbücher...

1. Welche Arbeitsfelder bedient meine Einsatzstelle?

2. Welche Klienten befinden sich in meiner Einsatzstelle (Anzahl/Alter/Herkunft)?

3. Welche besonderen Aktionen fanden in der Vergangenheit einmalig oder regelmäßig statt?

4. Was könnte der Zielgruppe/den Klienten in Zukunft gefallen bzw. was ist sinnvoll?

5. Wo möchte ich mich ausprobieren - Altbewährtes oder Neues?

6. Welchen Mehrwert hat dies für die einzelnen Beteiligten?

7. Welche persönlichen Kompetenzen/Fähigkeiten kann ich einbringen?

8. Wen kann ich als erfahrenen Mitarbeiter/Anleiter/Mentor einbeziehen?

OFFENE FRAGEN:

\section{PROJEKTSKIZZE:}

1. Welchen Namen hat das Projekt und wie kann ich es kurz beschreiben und bewerben? Welches Alternativprojekt habe ich?

2. Zeitmanagement - was soll wann erledigt werden und wie viel Zeit benötigt dies? Bitte entwerfen Sie in kurzes Skript, wann welcher Arbeitsschritt getan werden muss. (Kleiner Tipp: je vorbereiteter, desto besser läuft das Projekt.)

3. Welche Materialien/Räumlichkeiten werden benötigt?

4. Finanzierung - wie viel Kostet das Projekt und woher erhalte ich das Budget dafür? (Von der Einsatzstelle, vom Träger, von der Zielgruppe als Eigenbeitrag?)

5. Welche Personen helfen mir bei Planung, Durchführung, Präsentation? (Z.B. eine weitere FSJ-Kraft, Anleiter, Vorgesetzte, Einzelpersonen aus der Zielgruppe?)

6. Wie kann ich den Projektverlauf dokumentieren und abschließend kurz präsentieren? 
Die Projektmethode zielt damit auf eine engere Kooperation zwischen Träger, Einsatzstelle, Freiwilligen und Klienten, wie sie in Abbildung 3 überblicksartig dargestellt ist.

\section{Abbildung 3: Bereiche und Zuständigkeiten der Projektmethode}

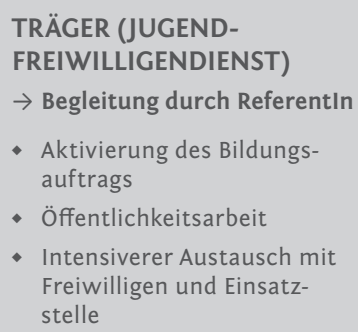

$\rightarrow$ Begleitung durch Referentln

- Aktivierung des Bildungsauftrags

- Öffentlichkeitsarbeit

- Intensiverer Austausch mit Freiwilligen und Einsatzstelle
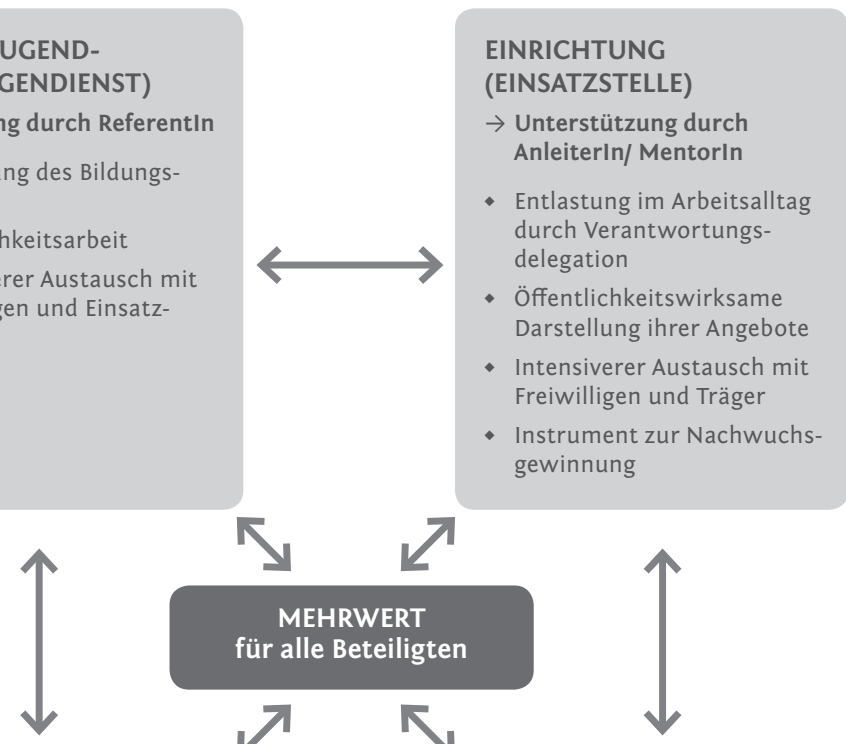

\section{MEHRWERT}

für alle Beteiligten

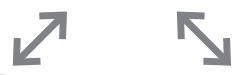

\section{EINRICHTUNC}

(EINSATZSTELLE)

$\rightarrow$ Unterstützung durch Anleiterin/Mentorin

Entlastung im Arbeitsalltag durch Verantwortungsdelegation

Öffentlichkeitswirksame

Intensiverer Austausch mit gewinnung

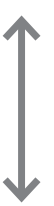

\section{FREIWILLIGE/R}

(PROJEKTLEITUNG)

$\rightarrow$ Verantwortliche/r

- Persönlichkeitsentwicklung bzgl. Selbstvertrauen, Verantwortungsbewusstsein, Organisationsfähigkeit

- Intensiverer Kontakt zu Klienten, Anleitung, ReferentIn und anderen Freiwilligen

- Selbstwirksamkeit

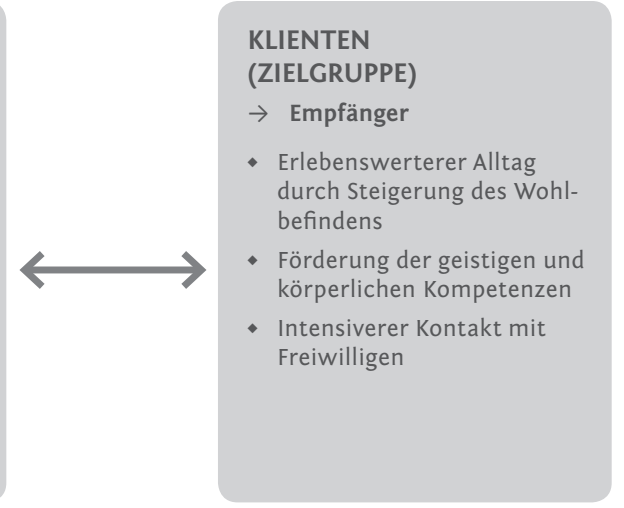




\section{Ein Beispiel aus der Praxis}

Im Folgenden beschreiben wir ein Beispiel für die Umsetzung eines solchen Projektauftrags, der im Rahmen des Bildungsauftrags der Freiwilligen Sozialen Dienste Nordbayern erteilt worden ist. ${ }^{17}$ Durchgeführt wurde das Projekt von zwei Freiwilligen, die ihr Freiwilliges Soziales Jahr im Bereich der Altenhilfe nach § 71 SGB XII abgeleistet haben. Der Leistungsauftrag der Altenhilfe beinhaltet, „Schwierigkeiten, die durch das Alter entstehen, zu verhüten, zu überwinden oder zu mildern und alten Menschen die Möglichkeit zu erhalten, am Leben in der Gemeinschaft teilzunehmen." 18 Gewährt werden u. a. Leistungen zu einer Betätigung und zum gesellschaftlichen Engagement, wenn sie vom alten Menschen gewünscht wird, Leistungen zum Besuch von Veranstaltungen oder Einrichtungen, die der Geselligkeit, der Unterhaltung, der Bildung oder den kulturellen Bedürfnissen alter Menschen dienen bzw. Leistungen, die alten Menschen die Verbindung mit nahe stehenden Personen ermöglichen. ${ }^{19}$

Eingesetzt waren die Freiwilligen in der Tagespflege „Im Kreuz zu Haus“ der Evangelisch-Lutherischen Kirchengemeinde Kreuzkirche in Bayreuth. Dort werden Hilfeleistungen für alte Menschen angeboten, „die Betreuung, Versorgung oder Pflege benötigen, aber in ihrer vertrauten häuslichen Umgebung wohnen bleiben möchten. “ ${ }^{20}$ Den Pflegebedürftigen soll einerseits die vertraute Umgebung und die häusliche Gemeinschaft so lange wie möglich erhalten bleiben, andererseits sollen die Familienangehörigen bei der Pflege unterstützt, begleitet und entlastet werden. Das Ziel der Einrichtung besteht in der Erhaltung der Selbstständigkeit: Um vorhandene Fähigkeiten zu erhalten und verloren gegangene wieder zu aktivieren, wird ein individuelles Beschäftigungsprogramm mit tagesstrukturierenden Maßnahmen angeboten, das jedem Gast ermöglicht, sich sinnvoll zu beteiligen bzw. dabei zu sein. Intendiert ist, dass sich Menschen mit ihren Möglichkeiten und Grenzen annehmen und entwickeln. Daher gilt es, Lebensperspektiven zu erhalten und durch die pflegerische Unterstützung einen Weg zu bereiten, die häusliche Umgebung nicht aufgeben zu müssen.

$\mathrm{Zu}$ den Pflegeleistungen gehören die Sicherung der ärztlichen Behandlung, die Unterstützung bei der Essenseinnahme sowie die Erhaltung und Aktivierung der individuellen Mobilität. Ein besonderer Schwerpunkt der Einrichtung liegt auf

17 Das beschriebene Projekt ist der Broschüre „Es kommt nicht darauf an, wie alt man wird, sondern wie man alt wird“ entnommen (Zitat Prof. Dr. Ursula Lehr, ehem. Bundesfamilienministerin, Vorsitzende der Bundesarbeitsgemeinschaft der Senioren-Organisationen, BAGSO), herausgegeben von: Freiwillige Soziale Dienste (FSD) Nordbayern der Evangelischen Jugend Nürnberg, 2013 (die Broschüre ist einsehbar auf der Webseite: www.fsd-nordbayern.de).

18 \1, Abs. 12 Das Zwölfte Buch Sozialgesetzbuch - Sozialhilfe - (Artikel 1 des Gesetzes vom 27. Dezember 2003, BGBI. I S. 3022, 3023), das zuletzt durch Artikel 1 des Gesetzes vom 1. Oktober 2013 (BGBI. I S. 3733) geändert worden ist.

19 Ebd., Abs. 2

20 http://www.im-kreuz-zu-haus.de/ueberuns.html 
der Erinnerungsarbeit, durch die bei hochbetagten bzw. demenzerkrankten Menschen dem ,Zerfall' der persönlichen Erinnerung vorgebeugt werden soll. „Das Besinnen auf Erfolge, das Erreichte, das Geleistete in Beruf und Privatleben können Selbstbewusstsein, Selbstwertgefühl und Selbstachtung stärken.“ ${ }^{21}$ Für eine solche Erinnerungsarbeit eignen sich z. B. Gegenstände, die einen Bezug zu früher herstellen (z. B. alte Werkzeuge, Haushaltsgeräte, Bücher, Zeitungsartikel, Musik usw.), aber auch der Besuch von vertrauten Orten kann eine ,Brücke in die Vergangenheit' bauen.

In diesem Kontext siedelten die Freiwilligen ihr Projekt an. Mit Unterstützung der Einrichtungsleitung organisierten sie einen Tagesausflug in die Bayreuther Eremitage, einem Ort, der vielen Gästen der Tagespflege aus ihrem Leben bekannt ist. Neben einem Spaziergang durch die Parkanlage engagierten die Freiwilligen eine Märchenerzählerin, die den teilweise dementiell erkrankten Gästen etwas von ihren Erinnerungen zurückbringen sollte.

\section{Erste Erfahrungen und Ausblicke}

Eine systematische Evaluation zur hier dargestellten Projektmethode steht zwar noch aus, als erste Erfahrung lässt sich jedoch festhalten, dass Freiwillige vom motivierenden und lernfördernden Charakter der Methode berichten, vor allem im Hinblick auf ihren hohen Grad an Aktivierung eigener Fähigkeiten und Interessen, dem eigenständigen Zeitmanagement und der offenen Themenwahl. Dieser positive Effekt konnte im Übrigen auch bei den Einsatzstellen beobachtet werden. Während als Mehrwert für die Freiwilligen eine positive Erfahrung von Selbstwirksamkeit, ein wachsendes Selbstvertrauen und Verantwortungsbewusstsein bzw. ein intensiverer und engerer Kontakt zur Einrichtung (Kollegenschaft, Klientel) verbucht werden kann, wurde von Seiten der Anleiter und Anleiterinnen in den Einrichtungen eine wesentliche Entlastung im Arbeitsalltag durch Verantwortungsdelegation, ein intensiverer Austausch mit Freiwilligen und Träger sowie ein besserer Kontakt zwischen Freiwilligen und Klientel berichtet.

Allerdings ergeben sich im Hinblick auf die oben dargestellte intentionale Förderung des Nicht-Intentionalen im Kontext von Kompetenzorientierung, Lernformen und Alltagsbildung aus unserer Sicht vier weiterführende Fragen bzw. Forschungsbedarfe.

1. Im pädagogischen Kompetenzverständnis wird grundsätzlich davon ausgegangen, dass Kompetenzen erlernbar sind bzw. verstärkt werden können. Das heißt vor allem, dass sie von den Einsatzstellen nicht vorausgesetzt werden können, sondern vielmehr erst im Laufe des Arbeitsprozesses entwickelt oder

21 http://www.im-kreuz-zu-haus.de/ueberuns.html 
differenziert werden. Dass dabei die funktionalen Anforderungen der Arbeitswelt in den Einsatzstellen und ihren funktional-differenzierten Arbeitsabläufen in einem grundsätzlichen Spannungsfeld mit den Lernvoraussetzungen und Entwicklungsbedürfnissen der Lernenden stehen, stellt eine zentrale Problematik der Konkretisierung des Bildungsauftrag der Jugendfreiwilligendiensten dar (Klieme 2004). Aus der Perspektive der konzeptionellen Verfasstheit von Jugendfreiwilligendienste ist zu betonen, dass insbesondere die Einsatzstellen die Strukturen dafür bereitstellen müssen, dass der Kompetenzerwerb durch die Freiwilligen überhaupt möglich ist. Vor diesem Hintergrund ist zu betonen, dass ,Kompetenzen“ zunächst einmal nichts anderes darstellen als theoretische Konstrukte (Hartig 2008). Sie spiegeln keine objektiven Sachverhalte wider, sondern sind abhängig von Zuschreibungen unterschiedlichster Akteure. Ihre Definition (und ihre Messung) erfolgt daher immer wertgebunden. Aus dieser Perspektive ist es notwendig, empirisch die Frage zu klären, an welchen Kompetenzen sich Einsatzstellen in ihrer Arbeit überhaupt orientieren, inwieweit diese mit den Kompetenzbereichen des DeSeCo übereinstimmen und inwiefern die Definition und Überprüfung von Kompetenzen mit dem Bildungsauftrag der Jugendfreiwilligendienste in Einklang zu bringen ist?

2. Im allgemeinen Verständnis von Kompetenzorientierung ist der Erwerb von Kompetenzen immer auf ein bestimmtes zu erreichendes Ergebnis bezogen. Weder sind also die Definition von Kompetenzen beliebig noch die Formen ihres Erwerbs. Gerade weil im nicht-intentionalen, alltagsbildenden Setting von Einsatzstellen unzweifelhaft Kompetenzen erworben werden, ergibt sich als zentrale Herausforderung die Aufgabe, den Erwerb solcher engagementspezifischer Kompetenzen im Jugendfreiwilligendienst leistungsbezogen zu erfassen und zu reflektieren (Klieme u. a. 2003: 71ff.). Es stellt sich daher die Frage, wie der Kompetenzerwerb in einem non-formalen Bildungssetting angesichts nicht-intentionaler Lernprozesse überhaupt festgestellt werden kann? Einen geeigneten Anknüpfungspunkt hierfür bietet sicherlich die Evaluation von Freiwilligen-Projekten wie sie hier als informelle Lernform im nicht-formalen Bildungssetting beschrieben wurden. Bei der weiteren Projektauswertung wäre dabei vor allem auf die subjektive Bedeutung von Prozessen des Kompetenzerwerbs abzuheben, die in der Selbstbeschreibung der Freiwilligen ihren Niederschlag findet.

3. Der Befund aus der allgemeinen Studie zum Kompetenzerwerb im freiwilligen Engagement verweist allerdings auch auf die Grenzen der Projektmethode. Diese offene, nicht-intentionale Form des Lernens dürfte sich dann als schwierig bzw. Überforderung herausstellen, wenn Lernende kaum Selbstwirksamkeitserfahrungen im Hinblick auf selbstorganisierte Lernprozesse mitbringen, u. U. Ängste und Hemmungen aber auch Lernschwächen vorhanden sind oder 
andere psychische Störungen negative Lernerfahrungen verstärken. Der Kompetenzerwerb dürfte dadurch erheblich erschwert oder gar unmöglich sein. Damit in engem Zusammenhang steht, dass mit der Projektmethode eine spezifische Art des Lernens bzw. von Handlungskompetenz vorausgesetzt wird. Als Hypothese für die Evaluation kann dabei davon ausgegangen werden, dass sich für den Kompetenzerwerb im Jugendfreiwilligendienst für unterschiedliche Tätigkeitstypen unterschiedliche Lerngewinne der Akteure ergeben (Düx u. a. 2009). In einer Studie zum freiwilligem Engagement allgemein konnten Düx u. a. zeigen, dass sich freiwilliges Engagement zwar „positiv auf die Bandbreite an Erfahrungen und Kompetenzen aller Befragten auswirk[e]“" (ebd.: 266), allerdings die „Kompetenzgewinne ungleich auf verschiedene Gruppen verteilt" (ebd.) sind. Düx u.a. unterscheiden vier solcher Typen bzw. Profile: a) der Tätigkeitstyp des ,Organisierens‘ mit dem Tätigkeitsprofil ,Organisator'; b) ,Gruppenarbeit und Training mit Kindern und Jugendlichen' und ,Gruppenleiter/Trainer'; c) ,Arbeit in Ausschüssen und Gremien“ und ,Funktionär'; d) ,handwerklich-technische Arbeiten und praktische Hilfeleistungen und ,praktischer Helfer'. Entsprechend dürfte Kompetenzerwerb an den Typ der ausgeübten Tätigkeit bzw. das Tätigkeitsfeld gebunden sein. So „beschreiben die Tätigkeitstypen ,Funktionäre' und ,Organisatoren' durch ihre Aktivitäten im Engagement sowohl insgesamt als auch besonders in den Bereichen Organisation und Leitung größere und breitere Erfahrungs- und Kompetenzgewinne sowie stärkere Förderung durch das Engagement als ,Gruppenleiter und ,praktische Helfer““ (ebd.). Die Typen ,praktischer Helfer‘ und ,Gruppenleiter' können dementsprechend als ,Spezialisten' gekennzeichnet werden, die entlang der Tätigkeitsmerkmale ,helfen' bzw. ,leiten' sehr funktionsspezifische Kompetenzen und Kenntnisse erwerben, die Tätigkeitstypen, Organisator ' und ,Funktionär‘ dagegen als ,Generalisten', die vielfältige Kompetenzen in großem Umfang und vielen unterschiedlichen Bereichen gewinnen und entwickeln. Als Filter wirken dabei auch hier die aus den Schulleistungsstudien bekannten Faktoren wie Schulbildung, Herkunft und Geschlecht. So kommen Düx u.a. zu dem Ergebnis, dass „Frauen, Migranten, Personen mit niedriger Schulbildung sowie Engagierte aus der ehemaligen DDR ihrem jugendlichen Engagement weniger Lernchancen und Kompetenzgewinnen ... [zuschreiben] als die anderen Engagierten“ (ebd.). Inwiefern solche Erfahrungen das Lernen und Arbeiten in den Einsatzstellen beeinflussen, ist bislang ein Forschungsdesiderat. Die Erfahrung des, langen Arms der Schule' mit ihren Momenten des Scheiterns, aber auch der erfolgreichen Bewältigung von schulischen Herausforderungen und Ansprüchen liegen in der Regel nur wenige Wochen und Monate zurück und dürften das engagementspezifische Lernen erheblich beeinflussen. Insofern stellt sich die Frage, inwiefern in den Jugendfreiwilligendiensten an schulische Lern(miss)erfolge angeknüpft werden kann bzw. muss. Wie also 
gelingt - angesichts negativer oder positiver, formeller Lernerfahrungen - der intendierte Erwerb der Schlüsselkompetenzen, wie sie im DeSeCo beschrieben sind? Die Differenzierung von Tätigkeitstypen und Tätigkeitsprofilen für das engagementspezifische Lernen könnte dabei als Heuristik für die Forschung über den Zusammenhang von informellen Lernprozesse im non-formalen Bildungssetting des Jugendfreiwilligendienstes mit den Erfahrungen der Freiwilligen in und mit formalen Bildungssettings und den entsprechenden formellen Lernprozessen dienen. Die Lernressourcen dürften also aufgrund der unterschiedlichen Bildungs- und Lernerfahrungen der heterogenen Lerngruppen in sehr unterschiedlichem Maße zu Verfügung stehen. Mit dem Ausgleich solcher ungleichen Ausgangschancen im Hinblick auf eine erfolgreiche Bewältigung des Projektauftrages ist eine besondere Herausforderung für den Auftrag an die Träger von Jugendfreiwilligendiensten verbunden, die Bildungsfähigkeit der Subjekte zu fördern. Es muss daher ein besonderes Augenmerk darauf gelegt werden, frühzeitig solche Lernschwierigkeiten zu erkennen und entsprechend zu intervenieren, um das intendierte nicht-intentionale Lernen zu unterstützen.

4. Eine letzte Herausforderung betrifft schließlich die Anschlussmöglichkeiten für solche informellen Lernprozesse auf der institutionellen Ebene derjenigen Bildungsinstitutionen, die Freiwillige nach dem Freiwilligendienst aufsuchen (also im Dualen System bzw. im Hochschulbereich). Auf theoretischer Ebene lässt sich die Bedeutung der Kompetenzorientierung im Jugendfreiwilligendienst einstweilen darin sehen, dass informelle Lernprozesse als eine zentrale Ressource für weitere schulische und außerschulische (formelle wie informelle) Lernprozesse im Lebenslauf betrachtet werden können. Inwieweit die berufliche bzw. universitäre Ausbildung an solche im Freiwilligendienst erworbenen Kompetenzen überhaupt anknüpft bzw. inwiefern die Jugendlichen/jungen Erwachsenen erworbene Kompetenzen in Ausbildung und Studium vertiefen können - auch das ist bislang ein Forschungsdesiderat.

\section{Literaturverzeichnis}

Arnold, Rolf und Ingeborg Schüßler (2001): Entwicklung des Kompetenzbegriffs und seine Bedeutung für die Berufsbildung und für die Berufsbildungsforschung, in: Komplexität und Kompetenz. Ausgewählte Fragen der Kompetenzforschung, hrsg. von Guido Franke, Bielefeld, S. 52-75.

Bundesministerium für Familie, Senioren, Frauen und Jugend (2001): Zukunftsfähigkeit sichern! Für ein neues Verhältnis von Bildung und Jugendhilfe, http://www.bmfsff.de/ RedaktionBMFSFJ/Broschuerenstelle/Pdf-Anlagen/PRM-15360-Umschlag-BroschureZukunftsfah.pdf (Zugriff am 8. Mai 2014).

Bundesministerium für Familie, Senioren, Frauen und Jugend (2005): Zwölfter Kinder und Jugendbericht: Berichte über die Lebenssituation junger Menschen und die Leistungen der Kinder - und Jugendhilfe in Deutschland. Bildung, Betreuung und Erziehung vor und neben der Schule, Berlin. 
Deutscher Städtetag (2007): Aachener Erklärung des Deutschen Städtetags anlässlich des Kongresses „Bildung in der Stadt“ am 22./23.11.2007, http://www.staedtetag.de/imperia/ md/content/veranstalt/2007/58.pdf (Zugriff am 8. Mai 2014).

Düx, Wiebken u.a. (2009): Kompetenzerwerb im freiwilligen Engagement. Eine empirische Studie zum informellen Lernen im Jugendalter, Wiesbaden.

Frey, Karl (2012/1982): Die Projektmethode. ,Der Weg zum bildenden Tun', Weinheim, Basel.

Hartig, Johannes (2008): Kompetenzen als Ergebnisse von Bildungsprozessen, in: Kompetenzerfassung in pädagogischen Handlungsfeldern. Theorien, Konzepte und Methoden, hrsg von Nina Jude u.a., Bonn-Berlin.

Klieme, Eckhard (2003): Zur Entwicklung nationaler Bildungsstandards, Bonn, http://katalog. ub.uni-bamberg.de/InfoGuideClient.ubgsis/start.do?Login=igubgtb2\&Query=10= "BV019754474“" u. a. (Zugriff am 8. Mai 2014).

Klieme, Eckhard (2004): Was sind Kompetenzen und wie lassen sie sich messen?, in: Pädagogik 6, S. 10-13.

Kultusministerkonferenz (2007): Handreichung für die Erarbeitung von Rahmenlehrplänen der Kultusministerkonferenz für den berufsbezogenen Unterricht in der Berufsschule und ihre Abstimmung mit Ausbildungsordnungen des Bundes für anerkannte Ausbildungsberufe, http://www.kmk.org/fileadmin/veroeffentlichungen_beschluesse/2007/2007_09_01Handreich-Rlpl-Berufsschule.pdf (Zugriff am 8. Mai 2014).

Langewand, Alfred (2002): Bildung. In: Erziehungswissenschaft, hrsg. von Dieter Lenzen, Reinbek, S. 69-98.

OECD (2005): Definition und Auswahl von Schlüsselkompetenzen, http://www.oecd.org/ pisa/35693281.pdf (Zugriff am 8. Mai 2014).

Rauschenbach, Thomas (2004): Non-formale und informelle Bildung im Kindes- und Jugendalter, Berlin, http://katalog.ub.uni-bamberg.de/InfoGuideClient.ubgsis/start.do?Login=igu bgtb2\&Query=10="BV019438716" (Zugriff am 08. Mai 2014).

Rauschenbach, Thomas (2007): Im Schatten der formalen Bildung. Alltagsbildung als Schlüsselfrage der Zukunft, in: Diskurs Kindheits- und Jugendforschung, Heft 4, S. 439-453.

Otto, Hans-Uwe und Thomas Rauschenbach (2008): Die andere Seite der Bildung. Zum Verhältnis von formellen und informellen Bildungsprozessen, Wiesbaden.

Rauschenbach, Thomas (2011): Alltagsbildung. Die andere Seite der Bildung, in: Bildung im Sport. Beiträge zu einer zeitgemäßen Bildungsdebatte, hrsg. von Michael Krüger und Nils Neuber, Wiesbaden, S. 35-52.

Rohlfs, Carsten, Marius Harring und Christian Palentien (2014): Kompetenz-Bildung. Soziale, emotionale und kommunikative Kompetenzen von Kindern und Jugendlichen, in: Kompetenz-Bildung.

Wischmeier, Inka und Hildegard Macha (2012): Außerschulische Jugendbildung, München.

Weber, Erich (1999): Pädagogik. Eine Einführung. Band I: Grundfragen und Grundbegriffe. Teil 3 Pädagogische Grundvorgänge und Zielvorstellungen - Erziehung und Gesellschaft/ Politik. 3 Bände. Donauwörth.

Weinert, Franz E. (2001): Leistungsmessungen in Schulen, Weinheim u.a. 